\title{
Contrast Enhancement with Oolong Tea in Staining of Ultrathin Sections
}

Ying Guo ${ }^{1}$, Reiner Bleher ${ }^{1,2,3}$ Shuyou $\mathrm{Li}^{1,3}$ and Vinayak P. Dravid ${ }^{1,3}$

1. NUANCE Center, Northwestern University, Evanston, IL 60208, USA, ${ }^{2}$ Chemistry of Life Processes Institute, Northwestern University, Evanston, IL 60208, USA, ${ }^{3}$. Department of Materials Science and Engineering, Northwestern University, Evanston, IL 60208, USA

Recently, several papers have been published on methodological studies of Oolong tea extract (OTE) staining of biological specimens in transmission electron microscopy (TEM). The use of OTE staining in biological sample preparation may enhance the contrast of TEM images as a substitute for uranyl acetate. The binding of contrasting moieties is affected by the fixative, embedding medium, the nature of the stain and the solvent, and also the concentration, temperature and $\mathrm{pH}$ of the staining solution [1]. In this study, our results indicate that the effect of Oolong tea staining is affected by reactive aldehyde groups in the specimens, resulting in improved membrane contrast.

Mouse ovary tissue was dissected into small pieces and fixed in $2.5 \%$ glutaraldehyde in $0.1 \mathrm{M}$ phosphate buffer, $\mathrm{pH} 7.4$ at room temperature for 2 hours. The specimens were dehydrated in a graded series of ethanol and embedded in Spurr resin. Ultrathin sections on grids were divided into two groups. All specimens were stained with 3\% Oolong tea (Oolong tea leaves, Harney \& Sons) in water with pH 7.4 for $20 \mathrm{~min}$, briefly rinsed with water, and stained with lead citrate for $3 \mathrm{~min}$. The protocols are summarized in Table 1.

\begin{tabular}{|l|l|}
\hline OTE + lead citrate & Glycine + OTE + lead citrate \\
\hline None & Incubated in 0.05M glycine for 5 min \\
\hline None & Washed with water briefly \\
\hline $\begin{array}{l}\text { Stained with 3\% Oolong tea for } 20 \mathrm{~min} \text { at room } \\
\text { temperature }\end{array}$ & $\begin{array}{l}\text { Stained with 3\% Oolong tea for 20 min at room } \\
\text { temperature }\end{array}$ \\
\hline Washed with water briefly & Washed with water briefly \\
\hline Stained with lead citrate for 3 min & Stained with lead citrate for 3 min \\
\hline Washed with water briefly twice & Washed with water briefly twice \\
\hline Airy dry & Air dry \\
\hline
\end{tabular}

Table 1. Incubation steps performed for staining sections with Oolong tea and lead citrate.

The TEM observation was carried out with a Hitachi HT-7700 S/TEM, operated at 80keV. In the samples stained with OTE and lead citrate, cytoplasmic components such as rough endoplasmic reticulum (rER) and mitochondria showed strong contrast and were clearly identified as reported in the literature [2]. When sections were incubated with glycine before staining with Oolong tea and lead citrate, the contrast of rER and mitochondria was faint and less clear compared to the previous sample. The mechanism of Oolong tea staining is not well understood and there are only a few explanations based on the reactions of phenolic groups in tannic acid staining [2]. From our experiments we conclude that the polyphenolic constituent in Oolong tea did not contribute to improved contrast when reactive aldehyde groups in the sample were inactivated by glycine. Theoretically, phenol is reactive towards formaldehyde at the ortho- and para sites, allowing up to 3 units of formaldehyde to attach to the ring structure: $\mathrm{HOC} 6 \mathrm{H} 5+\mathrm{CH} 2 \mathrm{O} \rightarrow \mathrm{HOC} 6 \mathrm{H} 4 \mathrm{CH} 2 \mathrm{OH}$. 
Therefore, contrast enhancement with oolong tea may be partially due to the fixatives used in the sample preparation.

\section{References:}

[1] K Yamaguchi et al, J. of Electron Microscopy 59 (2010), p. 113

[2] S Sato, A Adachi, Y Sasaki and M Ghazizadeh, J. of Microscopy, 229 (2008), p. 17.

[3] S Sato et al, Med Electron Microsc 36 (2003), p.179.
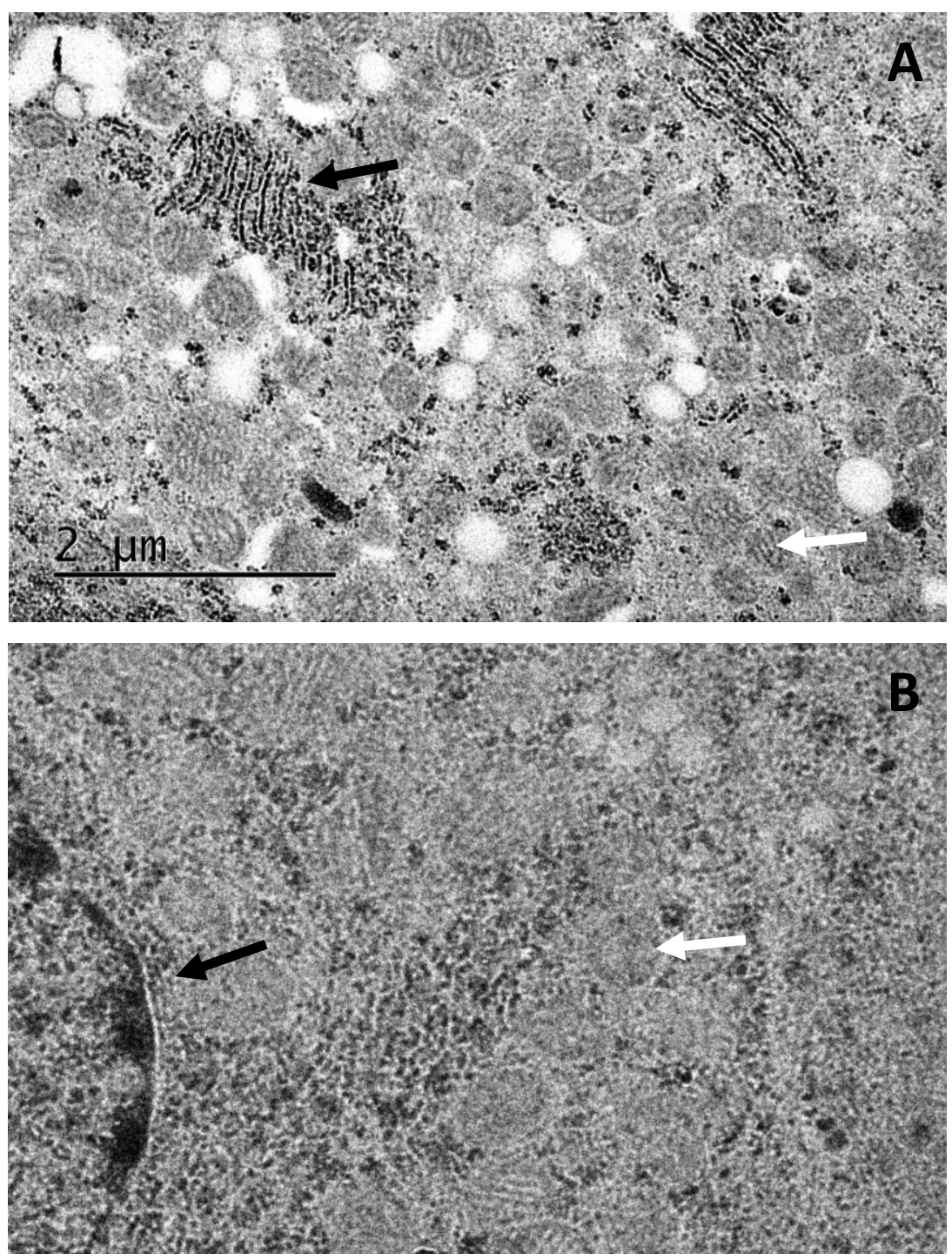

Figure 1. Sections of resin embedded mouse ovaries contrasted with (A) OTE and lead citrate, and (B) with glycine before contrasting with OTE and lead citrate. Mitochondriae (white arrows) and rER (black arrows) are well stained in (A), and show only weak contrast in (B). 